\title{
PROYECTO DE SEGURIDAD DEL PACIENTE TRAQUEOTOMIZADO PROCEDENTE DE UNA UNIDAD DE CUIDADOS CRÍTICOS
}

\section{Safety management of the patient with tracheostomy from a critical care unit}

\author{
Marleny CASASOLA-GIRÓN'; José Ignacio BENITO-OREJAS ${ }^{1}$; Felipe BOBILLO-DE LAMO²; \\ Laura PARRA-MORAIS²; Ramón CICUÉNDEZ-ÁVILA²; Darío MORAIS-PÉREZ1
}

SACYL. Hospital Clínico Universitario de Valladolid. ${ }^{1}$ Servicio de ORL. ${ }^{2}$ Servicio de Medicina Intensiva y Crítica. Valladolid. España.

Correspondencia: jibenito@ono.com

Fecha de recepción: 20 de agosto de 2017

Fecha de aceptación: 4 de octubre de 2017

Fecha de publicación: 7 de octubre de 2017

Fecha de publicación del fascículo: 1 de marzo de 2018

Conflicto de intereses: Los autores declaran no tener conflictos de intereses

Imágenes: Los autores declaran haber obtenido las imágenes con el permiso de los pacientes

Política de derechos y autoarchivo: se permite el autoarchivo de la versión post-print (SHERPA/RoMEO)

Licencia CC BY-NC-ND. Licencia Creative Commons Atribución-NoComercial-SinDerivar 4.0 Internacional

Universidad de Salamanca. Su comercialización está sujeta al permiso del editor

RESUMEN

PALABRAS CLAVE
Introducción y objetivo: La elevada morbimortalidad del paciente traqueotomizado que llega a una sala general procedente de la unidad de cuidados críticos, nos ha motivado a elaborar un proyecto de calidad y seguridad, con el objetivo de mejorar los cuidados y disminuir el número de incidentes que pudieran poner en peligro su vida. Método: Adaptando a nuestro medio las recomendaciones de la literatura, el programa se compone de cuatro elementos: información estandarizada, formación del personal implicado, seguimiento del paciente y régimen general. Resultados: La documentación realizada establece la manera de valorar a un enfermo con traqueotomía y de llevar a cabo su asistencia. A través de talleres interactivos se transmite la información al personal encargado de estos pacientes. El control periódico por un otorrinolaringólogo (ORL), una enfermera de $O R L$ y un médico intensivista, permite registrar la situación clínica y posibles complicaciones, aplicando protocolos específicos de decanulación y deglución. Finalmente se implantan un conjunto de normas generales, con el fin de disminuir la variabilidad. Discusión: El cuidado multidisciplinar en el paciente traqueotomizado es una intervención compleja donde la falta de tabulación previa, el importante número de enfermos neurocríticos, la multiplicidad de salas que pueden acoger a estos pacientes y su diversidad clínica, hacen difícil un seguimiento correcto. Conclusiones: Confiamos en que este proyecto logre sus objetivos, mejorando la calidad y seguridad del paciente portador de una cánula traqueal.

traqueostomía; traqueotomía; seguridad del paciente; mejora de la calidad; cuidados críticos; atención de enfermería; cuidados multidisciplinarios. 
SUMMARY

KEYWORDS

Introduction and objective: A patient with a tracheostomy has a high morbidity and mortality when comes to a general ward from the critical care unit. This situation has led us to develop a quality and safety program, to improve care and reduce the number of incidents that could endanger his life. Method: Adapting to our environment the recommendations of literature, the program is composed of four elements: standardized information, training of the staff involved, patient follow up and general scheme. Results: The elaborate documentation, offers the way of assessing a patient with tracheostomy, and carry out its assistance. Through interactive workshops, this information is transmitted to the staff responsible for these patients. The periodic inspection by an Otolaryngologist (ENT), an ENT nurse and an intensive care physician, allows to register the clinical situation and possible complications, applying specific protocols of decannulation and swallowing. Finally, we add a set of general rules, to decrease variability. Discussion: The multidisciplinary care in the patient with a tracheostomy is a complex intervention where the lack of previous data, the important number of neurocritical ill patients, the multiplicity of general wards that can accommodate these patients and its clinical diversity, make difficult proper monitoring. Conclusions: We are confident that this project can reach its goals, improving the quality and safety of patient carrier of a tracheal cannula.

tracheostomy; tracheotomy; safety patient; quality improvement; critical care; nursing care; multidisciplinary care

\section{INTRODUCCIÓN}

La elevada morbilidad y mortalidad (hasta de un $30 \%$ ) del paciente traqueotomizado que llega a una sala general desde las unidades de críticos (unidad de cuidados intensivos, reanimación, unidad de coronarias) [1-5], constituye uno de los motivos que justifica la existencia de servicios intermedios o equipos multidisciplinarios que favorezcan la progresiva evolución de estos enfermos [6-9]. La falta de protocolos estandarizados en el seguimiento del paciente portador de cánula traqueal $[10,11]$ y el desconocimiento de su asistencia fuera de las áreas especializadas [5, 9-10], condujo en diferentes hospitales del mundo, a la obtención de acuerdos para establecer una uniformidad de criterios en el cuidado de estos pacientes [12-16]. En 2013, la American Academy of Otolaryngology-Head and Neck Surgery reafirmó la importancia de una política común [17, 18]. Esta situación propició la formación de un equipo multidisciplinar en el Hospital Clínico Universitario de Valladolid (HCUV) que, tras configurar el proyecto de calidad y seguridad que presentamos en este trabajo, tuviera como objetivo fundamental, crear un ambiente seguro a estos enfermos, a fin de disminuir la incidencia de complicaciones.

\section{MATERIAL Y MÉTODO}

Tras obtener en 2014 la aceptación de la Comisión de Seguridad y Calidad del HCUV, de las diferentes direcciones sanitarias y de los jefes de las unidades implicadas, dimos comienzo a este proyecto. Adaptamos a nuestro medio las recomendaciones de la literatura, elaborando una documentación consensuada, sobre los cuidados del paciente portador de cánula traqueal, que fue incluida en la intranet del hospital, transmitiéndose de forma práctica al personal sanitario implicado. Se estableció una normativa común en los procedimientos y en la información que debía anotarse. Finalmente, los servicios de ORL y UCl iniciaron en 2015 un seguimiento y registro periódico de la evolución clínica de estos pacientes, determinando de acuerdo con el médico responsable, el momento de la decanulación y el inicio de la deglución, con la participación de la enfermería especializada de ORL y nutrición.

Para la gestión de los datos, elaboramos una base general de Microsoft Access y otra específica referida al proceso de decanulación y alimentación del paciente.

\section{RESULTADOS}

Este protocolo se fundamenta en 4 elementos: información estandarizada, formación del personal implicado, seguimiento del paciente y régimen general.

\section{INFORMACIÓN}

A partir de la revisión bibliográfica [15, 17-25] confeccionamos una amplia documentación que, bajo el epígrafe de «Guía de cuidados del paciente traqueotomizado", ha sido incluida en la intranet del hospital, para su rápido acceso. Tras exponer conceptos fundamentales, se define el objetivo de estos cuidados, que consiste en prevenir y disminuir las complicaciones y mejorar el manejo de las mismas en el paciente portador de cánula traqueal, para lo que es necesario: 
- Disponer del equipo suficiente, revisado periódicamente mediante listas de comprobación y localizado en un lugar fácilmente accesible.

- Vigilar la vía aérea, enseñando a toser al paciente, comprobando periódicamente la permeabilidad de la cánula, asegurando una suficiente hidratación y humidificación ambiental y sabiendo valorar la necesidad de aspiración.

- Evitar infecciones, aplicando una técnica estéril de aspiración, un correcto manejo del neumotaponamiento, un estoma limpio y seco, con curas diarias y recambio de apósitos siempre que sea preciso.

- Por último, prevenir, identificar y manejar complicaciones tales como, la presencia de un tapón, la decanulación accidental o la hemorragia.

En la información suministrada describimos también, cuándo, cómo y por qué llevar a cabo cada una de las maniobras necesarias en el mantenimiento de una cánula traqueal, enseñando el manejo de la aspiración, la limpieza de la cánula interna, el control del neumotaponamiento, el cambio de cánula traqueal y los cuidados del estoma. Un apartado especifica los signos de alarma de posibles complicaciones y las medidas a adoptar. A través de algoritmos, situados de manera visible en todas las salas hospitalarias que reciben a estos enfermos, se indican los pasos a seguir en caso de que se sospeche una parada cardio-respiratoria según sea el paciente portador de una traqueotomía o de una traqueostomía (laringectomía total) y a quién y cómo avisar en caso de urgencia [15].

Todo este material se resume en una "Guía práctica de actuación» en formato bolsillo, donde figuran en un tríptico las principales recomendaciones sobre el cuidado de la vía aérea y la actuación frente a complicaciones, en el paciente traqueotomizado, que el personal encargado puede llevar consigo.

\section{FORMACIÓN}

Desde mayo de 2015 y a través de la Comisión de Docencia del HCUV, se han organizado más de 30 talleres formativos para el personal de enfermería del hospital. En grupos reducidos de unas 6 personas, fueron impartidos por profesionales de enfermería expertos en el cuidado de estos pacientes tanto de la $\mathrm{UCl}$ como de ORL, con una duración aproximada de 1 hora y media. Se buscaba que los participantes interactuaran, utilizando para ello todo tipo de material educativo como, vídeos grabados directamente en las unidades de referencia, carteles explicativos, modelos de simulación para entrenamiento de las diferentes técnicas y toda la instrumentación habitual (cánulas de distintos tipos, aspiradores, medios de humidificación, manómetro...) (Figuras 1 y 2). Una breve base teórica [19] permitía comprender el porqué de estos cuidados, la diferencia de unos y otros pacientes en función de que se les hubiera realizado una traqueotomía o una traqueostomía, de que esta intervención se desarrollara de forma percutánea o quirúrgica y de los factores de riesgo acompañantes (cirugía reciente, obesidad, agitación, etc.).

Se destacaba la importancia de realizar al inicio de cada turno una serie de actuaciones sistematizadas, consistentes en: verificar la presencia del panel indicador situado en la cabecera de la cama del paciente, donde deben figurar los datos sobre fecha y tipo de traqueotomía, número y modelo de cánula..., y revisar la existencia y buen funcionamiento del material que debe estar en la habitación. Se comprueba a continuación la permeabilidad de la cánula interna (al menos una vez por turno y todas las que sean necesarias), la necesidad de aspiración, el control de presión del neumotaponamiento mediante manómetro, la buena sujeción de la cánula y la limpieza del apósito. Si el paciente tiene oxigenoterapia y humidificación, se confirma su correcta actividad. Finalmente se registran, en los programas informáticos correspondientes de seguimiento clínico, las maniobras efectuadas y todas las incidencias acaecidas. Así mismo, tras la llegada del paciente desde la unidad de críticos, se deben examinar, a través de la informatización hospitalaria, los datos enviados respecto a las características de la traqueotomía y los factores de riesgo del paciente.

Aunque esta formación general es fundamental para comprender los procedimientos y adquirir una cierta experiencia, los cambios de turno, las modificaciones de personal y la escasa frecuencia en algunas salas de pacientes canulados, obliga, como veremos en el siguiente apartado, a realizar un seguimiento general por personal experto que además se encargue de transmitir la información necesaria para el buen desarrollo de estas funciones. Esta constante relación, creemos que constituye el aspecto más importante en el adiestramiento del personal sanitario. 
Y como complemento, en aquellas unidades donde suele concentrarse un mayor número de pacientes con cánula traqueal (neurología, neurocirugía, neumología...), se organizan reuniones a demanda, donde se discuten problemas o se plantean actuaciones, que pretenden encontrar las mejores alternativas.

\section{SEGUIMIENTO DEL PACIENTE}

Desde julio de 2015 un médico intensivista y un médico ORL realizan un control periódico (1 o 2 veces a la semana en función de las necesidades) de los pacientes con cánula, ingresados en el hospital. En enero de 2017 se incorporó una enfermera, supervisora de la sala de ORL. Las funciones de este equipo multidisciplinar consisten en realizar un correcto seguimiento de los pacientes, atendiendo a su evolución y controlando los cuidados llevados desde la unidad, respecto a la correcta disposición de los paneles, del material, de la cánula y de los datos introducidos en los programas informáticos. Es también el momento de intercambiar información con el personal sanitario responsable y de resolver las dudas. En este sentido la participación de la enfermera ORL facilita la tramitación del material necesario y la solución a cuestiones prácticas en relación con los cuidados y la forma de organizarlos dentro de la sistemática particular de cada planta [26]. También se aprovecha para comentar con el médico responsable del paciente, el progreso del mismo y aquellos cambios que se consideren.

Se rellena una ficha de registro de cada paciente, con los datos más importantes en relación con su traqueotomía y los factores de riesgo añadidos, que se complementa con su situación a la salida de la unidad de críticos. También documentamos cada una de las visitas, respecto al estado de la cánula y su evolución, situación general del paciente, complicaciones y tipo de alimentación, apuntando si el material está dispuesto de forma adecuada en la habitación (Tabla 1). Estos datos son incluidos en una base de Microsoft Access, para su posterior análisis.

Hay dos procedimientos que en función de la progresión del proceso que sufre el paciente se intentan implementar cuanto antes, la decanulación y el tratamiento de la disfagia, si existe. La experiencia, durante los dos años de evolución del proyecto, nos ha permitido realizar adaptaciones y mejoras en la aplicación de estos protocolos, que actualmente desarrollamos de la forma siguiente.

Protocolo de decanulación: Variable, dependiendo del paciente y de la duración de la traqueotomía. Según los criterios generales establecidos en la bibliografía [27], el esquema que seguimos es el indicado en la Tabla 2. En la mayor parte de enfermos, pero especialmente en los neurocríticos, aplicamos además el protocolo endoscópico de Warnecke et al (2013) [28], donde una vez que se den las condiciones expuestas, se procede a la decanulación, siempre y cuando se compruebe mediante nasofibroscopia que:

- No hay aspiración de saliva.

- El paciente deglute de forma espontánea más de una vez por minuto, tras verificarlo al menos, durante 2 minutos.

- Existe sensibilidad laríngea, desencadenándose tos refleja al tocar con el extremo del fibroendoscopio el repliegue ariepiglótico de la laringe.

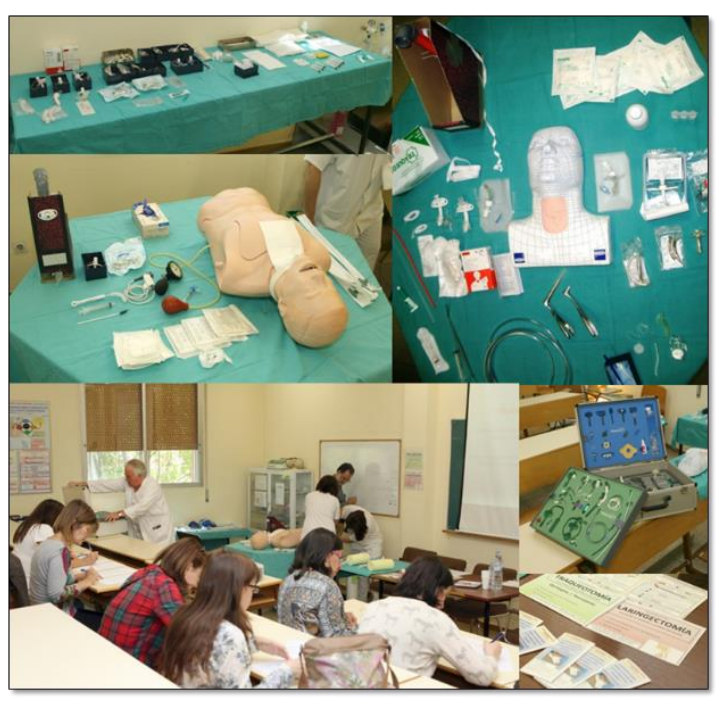

Figura 1. Taller formativo para profesionales de enfermería, sobre cuidados del paciente traqueotomizado, con parte del material utilizado. 


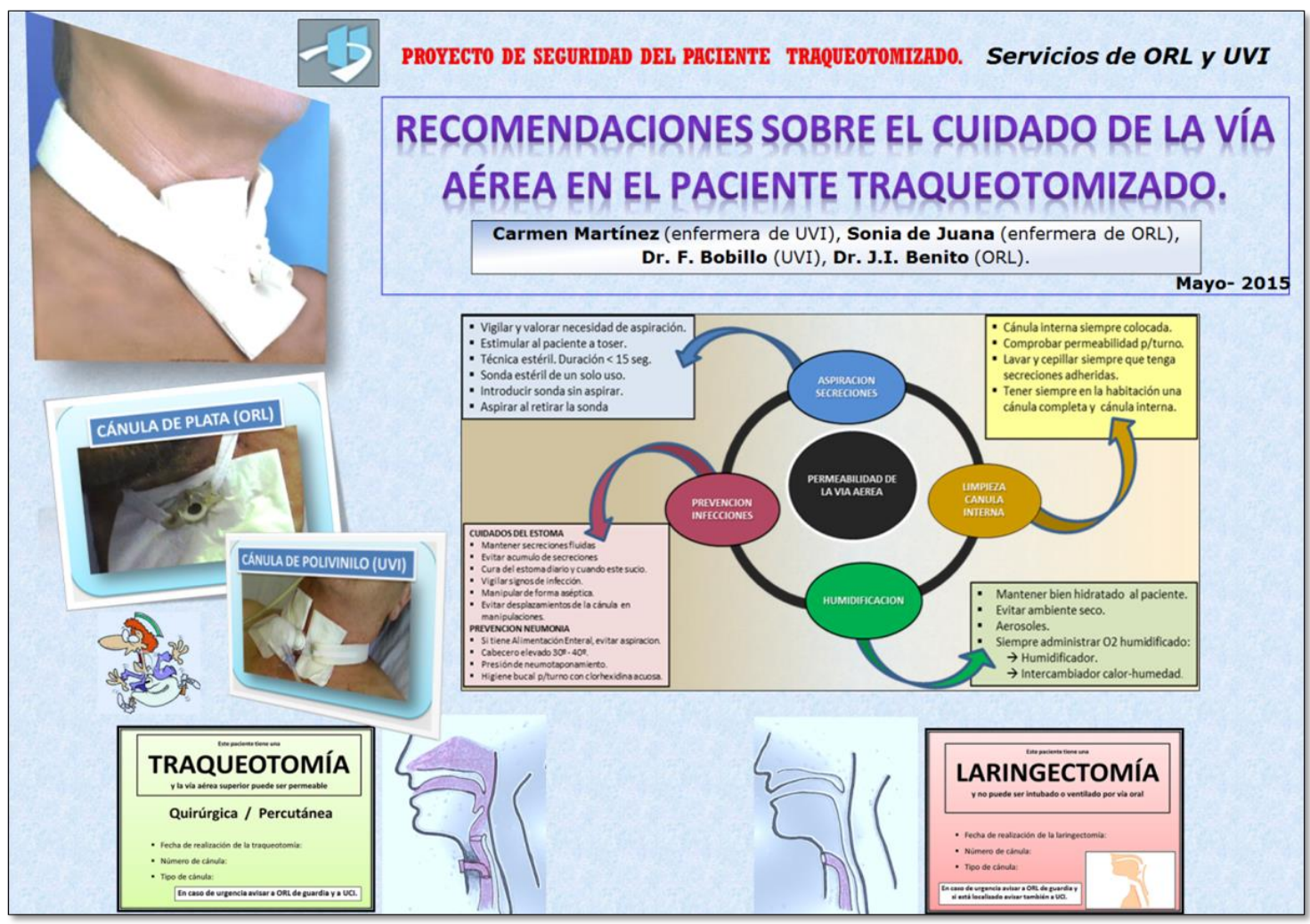

Figura 2. Panel informativo, utilizado en los cursos de formación, sobre distintos aspectos de la traqueotomía y de los cuidados que requiere el paciente portador de una cánula.

Satisfechos estos requisitos, aprovechamos la visión endoscópica, para ofrecer al paciente una cucharada de gelatina (preferiblemente de color rojo por su mejor visibilidad en caso de aspiración) y comprobar si existe o no una correcta deglución.

Sólo si se cumplen todos estos preceptos se continúa con el protocolo de deglución.

Los resultados de esta exploración también son introducidos en una base de datos de $\mathrm{Mi}$ crosoft Access.

Protocolo de deglución: Pretende constatar la capacidad de alimentación oral segura, en un paciente con cánula traqueal y neumotaponamiento.

Tras observar mediante endoscopia que la laringe es competente y cerciorarnos de la correcta deglución de gelatina, la enfermera de ORL contacta con el personal de enfermería del Servicio de Nutrición a fin de realizar un Test clínico de Volumen Viscosidad (TVV) [2930], que consiste en administrar volúmenes crecientes de diferentes consistencias a fin de detectar alteraciones de la seguridad o de la eficacia en la deglución [31]. Los signos que expresan una alteración de la seguridad son, la presencia de tos, el descenso superior a un $3 \%$ de la saturación de oxígeno (pulsioximetría) y cambios en la voz que sugieran ocupación laríngea. El residuo orofaríngeo y la deglución repetida serían los que indicarían una afectación de la eficacia [29]. Si se detecta un trastorno, se solicita la intervención de la Unidad de Disfagia, que evalúa el proceso mediante videoendoscopia. En todas aquellas enfermedades que son evolutivas, esta valoración se repite cuantas veces se considere necesario. 
Tabla 1. Ficha de registro con los datos del paciente, a su salida de la unidad de críticos. En la parte inferior del cuadro, se indica lo referido en cada una de las visitas posteriores.

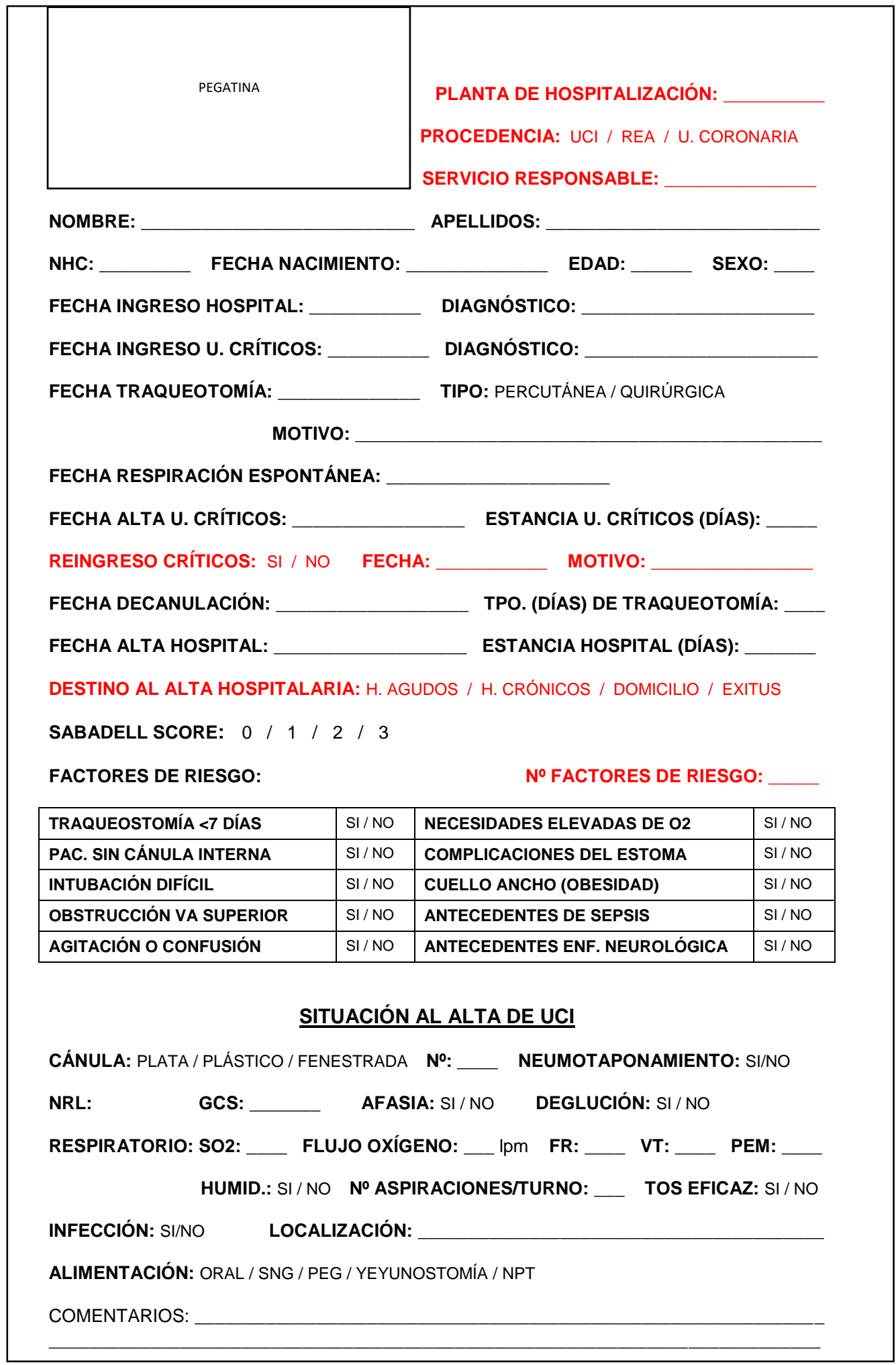


PROYECTO DE SEGURIDAD DEL PACIENTE TRAQUEOTOMIZADO PROCEDENTE DE UNA UNIDAD DE CUIDADOS CRíTICOS CASASOLA-GIRÓN M ET AL.

Tabla 2. Protocolo de decanulación en paciente con traqueotomía de larga duración por ventilación mecánica prolongada.

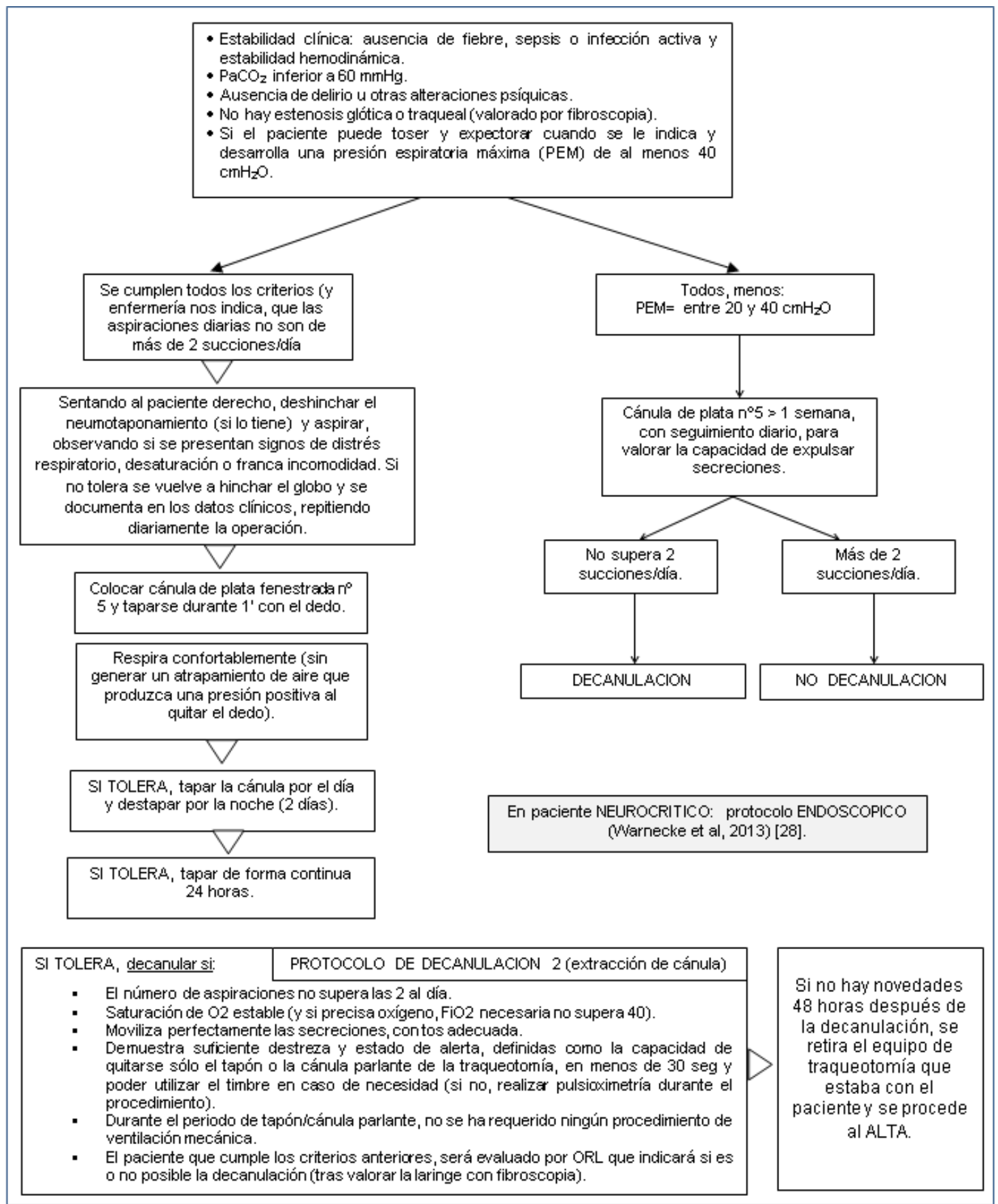

Las herramientas básicas de tratamiento son, la educación de los familiares y la adaptación de la dieta. El consejo terapéutico se basa en los siguientes principios [32]:

- Los pacientes con leves alteraciones de la eficacia deglutoria y una correcta seguridad van a poder seguir una dieta libre supervisada por su familia.

- En aquellos con alteraciones moderadas, se introducirán cambios dietéticos destinados a disminuir el volumen e incrementar la viscosidad del bolo. 
- Si la alteración es más severa se puede solicitar la competencia del Servicio de Rehabilitación, a fin de que la logopeda encargada de esta patología evalúe la conveniencia de un tratamiento rehabilitador.

- Finalmente, existe un grupo de pacientes en los que no es posible la alimentación oral, siendo necesaria la realización de una gastrostomía endoscópica percutánea (PEG), que en términos generales estaría indicada en:

- Pacientes inconscientes, con escasas posibilidades de recuperación, pero con esperanza de vida.

- Alteraciones severas de las fases oral o faríngea de la deglución y malnutrición.

- Alteraciones en la seguridad de la fase faríngea, sin respuesta al tratamiento rehabilitador, y

- En pacientes con importantes aspiraciones silentes.

De esta manera, la nutrición puede ser exclusivamente oral, oral adaptada o no oral. Mientras la deglución no sea segura, se mantiene la cánula con neumotaponamiento. Una vez decidida la forma de alimentación segura (oral enteral) se procede a la decanulación del paciente, siempre y cuando la situación respiratoria lo permita (Tabla 2). Aunque lo más habitual es que el inicio de la alimentación oral se acompañe de la decanulación, son posibles otras dos situaciones; la de un paciente decanulado y con PEG y la del que reinicia la deglución oral, pero no puede decanularse por insuficiencia respiratoria.

Desde la incorporación de la enfermera de ORL, además de controlar a los pacientes procedentes de las distintas unidades de críticos, también supervisamos a los que ingresan en el hospital con cánula traqueal, sea cual sea la causa, para verificar el buen uso de la misma y consensuar con la enfermería de planta los cuidados, necesidades y el correcto cumplimiento de los protocolos establecidos.

\section{RÉGIMEN GENERAL}

Se trata de ir creando normas comunes de aplicación global, para incrementar la seguridad. Entre las establecidas, destacamos las siguientes:

- El paciente saldrá de la unidad de críticos con una cánula de bajo riesgo (sin aspira- ción subglótica, con cánula interna...). Generalmente una cánula de polivinilo (PVC) con o sin neumotaponamiento, según necesidad.

- El traslado desde la unidad de críticos a la sala general se tratará de evitar en viernes y en fin de semana y se realizará por la mañana, intentando avisar de la llegada con una cierta antelación. En el registro informatizado del paciente, la enfermera encargada del alta de la unidad anotará todas las características del procedimiento y de la cánula, para que, a su llegada a planta, se reconozcan estas particularidades y el nivel de riesgo.

- Salvo en servicios especializados (ORL, UCl...) se utilizará el mismo tipo de cánula y la misma marca comercial en todo el hospital (cánula de polivinilo). Si el paciente puede prescindir del neumotaponamiento, se le colocará en caso de traqueotomía temporal, una cánula de plata fenestrada del №5, con cánula interna, antes de realizar la decanulación (Tabla 2).

- Los procedimientos utilizados en el cuidado de la cánula serán comunes, al igual que los equipos de oxígeno y humidificación que se utilicen en todo el hospital.

- Cada paciente tendrá un panel en la cabecera de su cama donde se indicarán los datos referentes a la traqueotomía o traqueostomía, la fecha de realización y las características de la cánula. Estos datos ofrecen al especialista un rápido control de las circunstancias, en caso de aviso urgente.

- En cada turno, el profesional de enfermería debe revisar el material de la habitación, la permeabilidad de la cánula interna y comprobar la sujeción de la cánula y la limpieza del apósito.

- Las salas que reciben a estos enfermos disponen de una caja, con todo el material necesario en caso de urgencia. En la tapadera, presenta un listado del contenido, que debe revisarse periódicamente. Suele disponerse junto al carro de paradas.

- El estoma se limpiará diariamente, siempre entre dos personas. Tras liberar la cánula de su sujeción al cuello, una de las profesionales sujeta y se responsabiliza de la cánula, mientras el compañero realiza la limpieza con suero fisiológico, anotando las alteraciones observadas. 
- La cánula traqueal, salvo excepciones, se cambia solamente una vez al mes por personal experto.

- Animamos continuamente a que los trabajadores sanitarios, refieran por escrito cualquier incidente acaecido en un paciente portador de cánula traqueal, con el fin de reforzar la eficacia de la seguridad [33].

\section{DISCUSIÓN}

La morbimortalidad del paciente traqueotomizado que llega a una sala general desde la $\mathrm{UCl}$, se agrava en nuestro medio por el importante porcentaje de enfermos con severas secuelas neurológicas - secundarias a traumatismo craneoencefálico (TCE) y accidente cerebral vascular agudo (ACVA), fundamentalmente- [34], a lo que se suma la debilidad muscular y la escasa capacidad de comunicación y colaboración. El cambio brusco que supone abandonar el paraguas de protección de la UCl, con monitorización y personal especializado las 24 horas, facilita el gran número de complicaciones que pueden presentarse al llegar a una sala convencional. Estas circunstancias, junto a la falta de protocolos estandarizados en el seguimiento de estos pacientes, condujeron a que en 2012 los Servicios de UCl y ORL del Hospital Clínico Universitario de Valladolid (HCUV), planificaran un proyecto formativo para profesionales de enfermería y técnicos en cuidados auxiliares de enfermería del hospital, a través de una serie de talleres prácticos. Tras este inicio, consideramos la conveniencia de estructurar un programa general hospitalario de calidad y seguridad para el paciente con cánula traqueal. Utilizando como guía el The national tracheostomy safety project (NTSP) del Reino Unido [4, 15], se puso en marcha en el año 2015.

Parece un problema generalmente aceptado, que a los sanitarios que trabajan fuera de las unidades especializadas, les falta conocimiento y destreza suficiente para tratar a pacientes con cánula traqueal [5, 10-11, 3, 36]. Sólo un pequeño porcentaje, ha recibido formación al respecto [5] y sin embargo se ha demostrado, que la aplicación de un módulo educativo estandarizado permite adquirir parte de estas habilidades y sobre todo incrementar la seguridad y confianza, al reducir la angustia que se suele sentir frente a estos enfermos $[10,16]$. Aunque se necesita mayor evidencia científica en cuanto a cómo deben ser los mejores cuidados en el paciente traqueotomizado [10], hay mucha literatura que refrenda los establecidos y se han publicado varios estándares de buenas prácticas [15, 17-25]. Creemos, por tanto, que la información que se transmite de manera muy interactiva a la plantilla sanitaria es suficiente, aunque dada la movilidad laboral, sería conveniente un reciclaje permanente. Este conocimiento teórico se traslada también de una manera práctica, a través de la colaboración del personal encargado de estos enfermos con los miembros del equipo multidisciplinar, que periódicamente supervisan la evolución de estos pacientes [8].

En las salas generales y sobre todo en las que tienen escasa afluencia de enfermos con cánula traqueal, la falta de protocolos, de planificación del seguimiento, la falsa creencia de competencia, el uso de diferentes procedimientos, el desconocimiento de determinados recursos, etc., incrementa el riesgo de incidentes $[5,8,10]$.

Mediante la creación de unidades intermedias o de grupos multidisciplinarios, se puede mejorar el resultado de este seguimiento $[6,7,9$, 12-14, 37-40]. El cuidado multidisciplinar es una intervención compleja y de difícil valoración debido a sus múltiples componentes, siendo discutible definir quién debe constituir este equipo [7]. Como en otros hospitales, consideramos imprescindible la presencia de un médico intensivista, que ha sido partícipe en muchos casos de la indicación y realización de la traqueotomía percutánea [5-9, 13, 37, 41]. Conoce al paciente, al médico responsable y puede valorar en qué momento se supera el motivo por el que se realizó la intervención. Un ORL experto en el manejo de este procedimiento, será el encargado de los primeros cambios de cánula, y de intentar resolver las complicaciones que pudieran surgir, además de determinar mediante fibroendoscopia el mejor momento de decanulación una vez estabilizados los factores generales. También creemos que la presencia de un profesional de enfermería competente resulta de gran ayuda en el control y resolución de todos aquellos factores intercurrentes, que se generan con el personal habitualmente encargado. Además, en nuestro medio, participa activamente en el proceso de decanulación, evaluación de la disfagia y en el control y seguimiento de los pacientes con cánula, que ingresan en el hospital por cualquier otro motivo. Pero mantenemos un permanente contacto 
con otras especialidades. De forma casi continua con logopedia y fisioterapia a través del Servicio de Rehabilitación, la Unidad de Disfagia del hospital, la enfermería de nutrición del Servicio de Endocrinología y Nutrición y con menos frecuencia, con los servicios de $\mathrm{Neu}$ mología, enfermedades infecciosas y todos aquellos, con los que, en función de las patologías del paciente, sea necesario coordinarse.

La uniformidad de criterios para reducir la variabilidad es una de las principales conclusiones obtenidas en consenso por la American Academy of Otolaryngology-Head and Neck Surgery [17-18]. Aplicando una normativa común, se ha intentado generalizar la forma de actuación, equiparándola en todo el hospital y simplificando al máximo el utillaje necesario, aunque existen situaciones complejas.

Uno de los pasos fundamentales en la recuperación del paciente traqueotomizado consiste en determinar el momento de la decanulación, pero las opiniones sobre cuál es el mejor procedimiento son variables y la decisión es complicada [10]. Hay pocos estudios que expresen la circunstancia idónea para decanular y están basados fundamentalmente en la experiencia personal de sus autores [42]. En base a la literatura publicada [8, 13, 27, 37, 43-47], aplicamos actualmente el protocolo de la Tabla 2 , junto a la evaluación fibroendoscópica ya descrita [28]. Pero nuestra mejor referencia es la que obtenemos de la evaluación continuada, de las posibles emergencias y del permanente contacto con el paciente.

Aunque el objetivo fundamental de este proyecto y de la constitución de un equipo multidisciplinar es intentar mejorar la calidad y seguridad del paciente con cánula, para disminuir el número de incidentes y muertes, es posible que se deriven otros beneficios. Varios autores afirman que desciende el número de readmisiones en $\mathrm{UCI}[12,16,39]$ y al seguir unos estrictos protocolos, se calcula que el periodo de decanulación se reduce hasta en un $20 \%$ [12, 13], lo que trae como consecuencia un acortamiento de la estancia hospitalaria [9, 12-14], suponiendo un gran ahorro para el sistema sanitario, dado el gasto que generan estos pacientes. Por otra parte, y para finalizar, el riesgo que entraña este procedimiento provoca litigios, en los que el ORL y los profesionales de enfermería son los más frecuentemente demandados, fundamentalmente por negligencia en los cuidados postoperatorios (81\%), lo que ratifica la importancia de un entrenamiento apropiado, de un registro de las Ediciones Universidad de Salamanca / CC BY-NC-ND [14] actuaciones y de la existencia de un equipo multidisciplinar organizado [48].

\section{CONCLUSIONES}

Esperamos que la aplicación de este proyecto mejore la calidad y seguridad del paciente portador de cánula traqueal, disminuyendo su morbimortalidad y de forma secundaria reduciendo el tiempo de decanulación y su estancia hospitalaria.

\section{AGRADECIMIENTOS}

A nuestra compañera Sonia de Juana Morrondo, supervisora de planta de ORL, porque su labor y experiencia, a pesar de la reciente incorporación al equipo, ya se han hecho imprescindibles. A todo el personal de enfermería y técnicos en cuidados auxiliares, que participan y aceptan con gusto nuestras indicaciones y a los diferentes servicios y estamentos hospitalarios por el apoyo que desde un principio nos han ofrecido. Ojalá algún día sean nuestros pacientes los agradecidos, porque significaría que valió la pena el esfuerzo de tantos.

\section{BIBLIOGRAFÍA}

1. Gordo $F$, Núñez A, Calvo E, Algora A. Mortalidad intrahospitalaria tras el alta de una unidad de cuidados intensivos en pacientes que han precisado ventilación mecánica. Med Clin (Barc). 2003; 121(7): 241-4.

2. Clec'h C, Alberti C, Vincent F, GarrousteOrgeas M, de Lassence A, Toledano D, et al. Tracheostomy does not improve the outcome of patients requiring prolonged mechanical ventilation: a propensity analysis. Crit Care Med. 2007;35(1):132-8.

3. Martinez $\mathrm{GH}$, Fernandez R, Casado MS, Cuena R, Lopez-Reina P, Zamora S, et al. Tracheostomy tube in place at intensive care unit discharge is associated with increased ward mortality. Respir Care. 2009;54(12):1644-52. 
4. McGrath BA, Thomas AN. Patient safety incidents associated with tracheostomies occurring in hospital wards: a review of reports to the UK National Patient Safety Agency. Postgrad Med J. 2010;86(1019):522-5.

5. Mondrup F, Skjelsager K, Madsen KR. Inadequate follow-up after tracheostomy and intensive care. Dan Med J. 2012;59(8):A4481.

6. Norwood MG, Spiers P, Bailiss J, Sayers RD. Evaluation of the role of a specialist tracheostomy service: from critical care to outreach and beyond. Postgrad Med J. 2004;80(946):478-80.

7. Garrubba M, Turner T, Grieveson C. Multidisciplinary care for tracheostomy patients: a systematic review. Crit Care. 2009;13(6):R177.

8. Wilcox SR, Schmidt UH. Are tracheostomized patients safe on regular hospital wards? Respir Care. 2009;54(12):1632-4.

9. Pandian V, Miller CR, Mirski MA, Schiavi AJ, Morad AH, Vaswani RS, et al. Multidisciplinary team approach in the management of tracheostomy patients. Otolaryngol Head Neck Surg. 2012;147(4):684-91.

10. Paul F. Tracheostomy care and management in general wards and community settings: literatura review. Nurs Crit Care. 2010;15(2):76-85.

11. Dorton LH, Lintzenich CR, Evans AK. Simulation model for tracheotomy education for primary health-care providers. Ann Otol Rhinol Laryngol. 2014;123(1):11-8.

12. Hettige $R$, Arora $A$, Ifeacho $S$, Narula $A$. Improving tracheostomy management through design, implementation and prospective audit of a care bundle: how we do it. Clin Otolaryngol. 2008;33, 488-91.

13. Cetto R, Arora A, Hettige R, Nel M, Benjamin L, Gomez CM, et al. Improving tracheostomy care: a prospective study of the multidisciplinary approach. Clin Otolaryngol. 2011;36:482-8.
14. Mirski MA, Pandian V, Bhatti N, Haut E, Feller-Kopman D, Morad A, et al. Safety, efficiency, and cost-effectiveness of a multidisciplinary percutaneous tracheostomy program. Crit Care Med. 2012;40(6):182734.

15. McGrath BA: Comprehensive tracheostomy care. The national tracheostomy safety project manual. Wiley Blackwell. 1ª Ed. 2014. pp 144.

16. Yelverton JC, Nguyen JH, Wan W, Kenerson MC, Schuman TA. Effectiveness of a standardized education process for tracheostomy care. Laryngoscope. 2015;125(2):342-7.

17. Mitchell RB, Hussey HM, Setzen G, Jacobs IN, Nussenbaum B, Dawson C, et al. Clinical consensus statement: tracheostomy care. Otolaryngol Head Neck Surg. 2013;148(1):620.

18. Benito-Orejas Jl. Consenso clínico en relación a los cuidados de la traqueotomía (artículo comentado). Rev ORL. 2014 [citado 23 Jul 2017]; 5(Supl.3):S1-8. Disponible en: https://gredos.usal.es/jspui/bitstream/10366/ 125011/1/revistaorl2014_supl3_traqueotomi a.pdf

19. Bobillo-De Lamo F, Benito-Orejas Jl, Martínez-Díez C, De Juana-Morrondo, MS. Cuidados del paciente traqueotomizado. Rev ORL. 2013 [citado 23 Jul 2017]; 4 (Supl.4): S1-18. Disponible en: https:/gredos.usal.es/jspui/bitstream/10366/ 124494/1/revistaorl2013_supl4_cuidadostra queotomia1.pdf

20. Pardal-Refoyo J. Guía de cuidados en Otorrinolaringología y Patología Cénvicofacial. Revista ORL. 2016 [citado 19 Jul 2017]; 8(1): 1-139. Disponible en: http://revistas.usal.es/index.php/24447986/article/view/13553

21. Clavel-Cerón R, Calvo-Torres MD, LuqueMartínez MJ, Subiela-García JA. Atención integral al paciente portador de cánula. Servicio Murciano de Salud. Murcia. $1^{\text {a }}$ Ed. 2016. [citado 19 Jul 2017]. pp 642. Disponible en:

http:/www.murciasalud.es/recursos/ficheros/ 367664-LIBRO_ENFERMERIA_W.pdf 
22. NHS Quality Improvement Scotland (NHS QIS). Caring for the patient with a tracheostomy. 2007 [citado 19 Jul 2017]. pp $47 . \quad$ Disponible en: file://C:/Users/OEM\%20USER/Downloads/ TRACHEOREV BPS MAR07.pdf

23. National Tracheostomy Safety Project. Information resource for the management of patients with tracheostomies and laryngectomies. 2010 [citado 19 Jul 2017]. pp 112. Disponible en: https://www.choiceforum.org/docs/natt.pdf

24. Agency for Clinical Innovation (ACl). Care of adult patients in acute care facilities with a tracheostomy. Clinical practice guideline. 2013 [citado 19 Jul 2017]. pp 101. Disponible en:

https://www.aci.health.nsw.gov.au/ data/as sets/pdf file/0005/181454/ACl_Tracheosto my_CPG.pdf

25. Intensive Care Society Council. Standards for the care of adult patients with a temporary Tracheostomy; STANDARDS AND GUIDELINES. 2014 [citado 19 Jul 2017]. pp 56. Disponible en: http://www.theawsomecourse.co.uk/ICS/ICS \%20Tracheostomy\%20standards\%20(2014 ).pdf

26. Peláez-Palacios MS, Fermoso-Palmero MJ. Investigación bibliográfica sobre cuidados de enfermería y seguridad en el paciente con traqueotomía. Rev Soc Otorrinolaringol Castilla Leon Cantab La Rioja. 2012 [citado 23 Jul 2017];5:55-65. Disponible en: https:/gredos.usal.es/jspui/bitstream/10366/ 124536/1/revistaorl201407_revisiontraqueot omia.pdf

27. Carretero-Rodríguez I, Pardal-Refoyo JL. Investigación bibliográfica sobre la decanulación en pacientes traqueotomizados. Rev Soc Otorrinolaringol Castilla Leon Cantab La Rioja. 2015 [citado 23 Jul 2017];6(20):160-170. Disponible en: https://gredos.usal.es/spui/bitstream/10366/ 125984/1/revistaorl201520_decanulacion.pd

28. Warnecke $\mathrm{T}$, Suntrup $\mathrm{S}$, Teismann $\mathbb{I K}$, Hamacher C, Oelenberg S, Dziewas R. Standardized endoscopic swallowing evaluation for tracheostomy decannulation in critically ill neurologic patients. Crit Care Med. 2013;41(7):1728-32.
29. Clavé $\mathrm{P}$, Arreola $\mathrm{V}$, Romea $\mathrm{M}$, Medina L, Palomera E, Serra-Prat M. Accuracy of the volume-viscosity swallow test for clinical screening of oropharyngeal dysphagia and aspiration. Clin Nutr. 2008;27(6):806-15.

30. Rofes L, Arreola V, Clavé P. The volumeviscosity swallow test for clinical screening of dysphagia and aspiration. Nestle Nutr Inst Workshop Ser. 2012;72:33-42.

31. García-Botrán B, Granell-Navarro J, Gutiérrez-Fonseca R, Barba-Martín R. Cribado clínico de la disfagia orofaríngea en un hospital general. Primeros resultados. Rev Soc Otorrinolaringol Castilla Leon Cantab La Rioja. 2014 [citado 23 Jul 2017];5(14):112123. Disponible en: https://gredos.usal.es/ispui/bitstream/10366/ 124545/1/revistaorl201414_cribadodisfagia. pdf

32. Clavé P, Terré R, de Kraa M, Serra M. Actitud a seguir ante una disfagia orofaríngea. Rev Esp Enferm Dig. 2004;96(2):119-131.

33. Pérez-Boillos M, Alcalde-Martín M, GarcíaPalomar I, González-Pastrana J, MonteroAlonso M, García-Espinosa P. Sistema de notificación de incidentes sin daño en el sistema de salud de Castilla y León. Revista ORL.2017 [citado 24 Jul 2017];8(1):17-21. Disponible en: http://revistas.usal.es/index.php/24447986/article/view/15423

34. Gandía-Martínez F, Martínez-Gil I, AndaluzOjeda D, Bobillo de Lamo F, Parra-Morais L, Díez-Gutiérrez F. Análisis de la traqueotomía precoz y su impacto sobre la incidencia de neumonía, consumo de recursos y mortalidad en pacientes neurocríticos. Neurocirugia (Astur). 2010 Jun;21(3):211-21.

35. Smith-Miller C. Graduate nurses' comfort and knowledge level regarding tracheostomy care. J Nurses Staff Dev. 2006;22(5):222-9; quiz 230-1.

36. Casserly P, Lang E, Fenton JE, Walsh M. Assessment of healthcare professionals' knowledge of managing emergency complications in patients with a tracheostomy. $\mathrm{Br} J$ Anaesth. 2007 Sep;99(3):380-3. 
37. Tobin AE, Santamaria JD. An intensivist-led tracheostomy review team is associated with shorter decannulation time and length of stay: a prospective cohort study. Crit Care. 2008;12(2):R48.

38. Arora $A$, Hettige $R$, Ifeacho $S$, Narula $A$. Driving standards in tracheostomy care: a preliminary communication of the St Mary's ENT-led multi disciplinary team approach. Clin Otolaryngol. 2008;33(6):596-9.

39. Sodhi K, Shrivastava A, Singla MK. Implications of dedicated tracheostomy care nurse program on outcomes. J Anesth. 2014;28(3):374-80.

40. de Mestral C, lqbal S, Fong N, LeBlanc J, Fata P, Razek T, et al. Impact of a specialized multidisciplinary tracheostomy team on tracheostomy care in critically ill patients. Can J Surg. 2011;54(3):167-72.

41. Choate K, Barbetti J, Sandford M. Tracheostomy: clinical practice and the formation of policy and guidelines. Aust Nurs J. 2003;10(8):17-9.

42. Pasqua F, Nardi I, Provenzano A, Mari A; Lazio Regional Section, Italian Association of Hospital Pulmonologists (AIPO). Weaning from tracheostomy in subjects undergoing pulmonary rehabilitation. Multidiscip Respir Med. 2015;27(10):35.
43. Ceriana $P$, Carlucci $A$, Navalesi $P$, Rampulla C, Delmastro M, Piaggi G, et al. Weaning from tracheotomy in long-term mechanically ventilated patients: feasibility of a decisional flowchart and clinical outcome. Intensive Care Med. 2003;29(5):845-8.

44. Dawson D. Essential principles: tracheostomy care in the adult patient. Nurs Crit Care. 2014;19(2):63-72.

45. Schmidt U, Hess D, Bittner E. To decannulate or not to decannulate: a combination of readiness for the floor and floor readiness?. Crit Care Med. 2011;39(10):2360-1.

46. Stelfox HT, Crimi C, Berra L, Noto A, Schmidt $\mathrm{U}$, Bigatello $L M$, et al. Determinants of tracheostomy decannulation: an international survey. Crit Care. 2008;12(1):R26.

47. O'Connor HH, Kirby KJ, Terrin N, Hill NS, White AC. Decannulation following tracheostomy for prolonged mechanical ventilation. J Intensive Care Med. 2009;24(3):187-94.

48. Farida JP, Lawrence LA, Svider PF, Shkoukani MA, Zuliani GF, Folbe AJ, et al. Protecting the airway and the physician: Aspects of litigation arising from tracheotomy. Head Neck. 2016;38(5):751-4. 